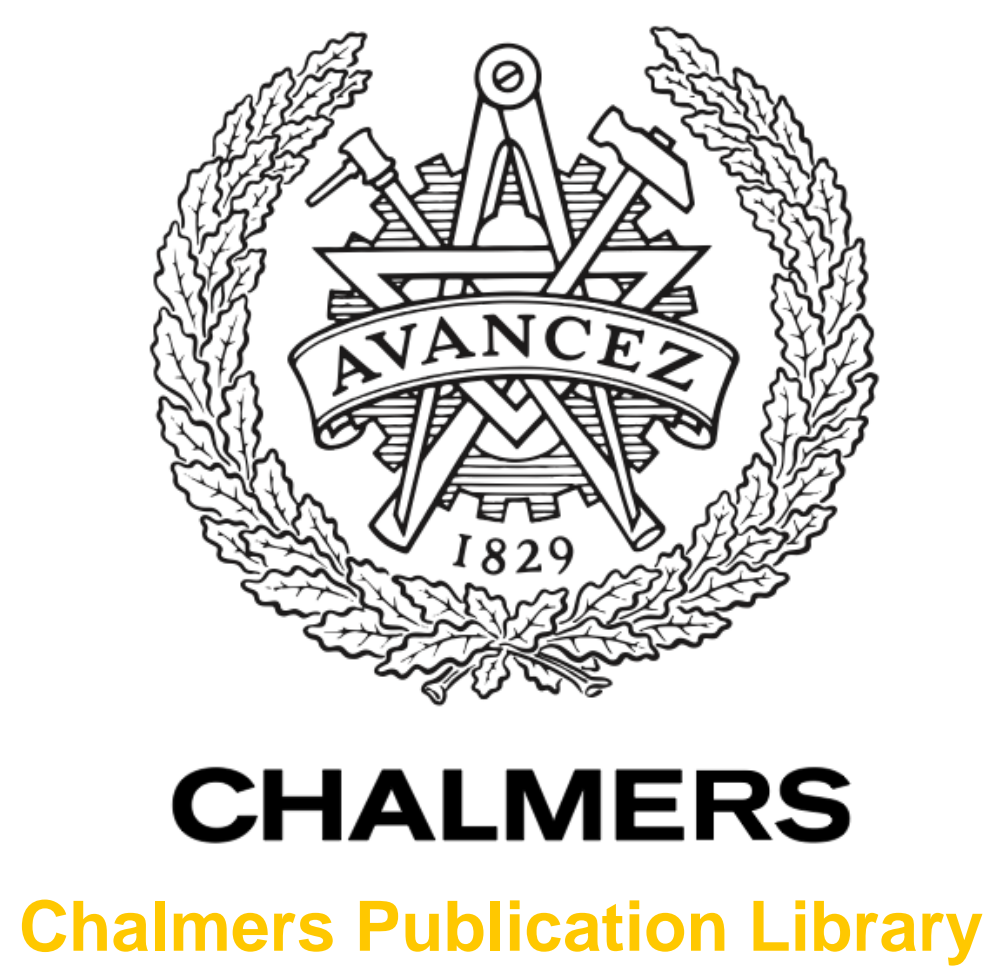

\title{
Composite Ferroelectric FBARs That Are Switchable Between the First and Second Harmonics: Experimental Demonstration
}

This document has been downloaded from Chalmers Publication Library (CPL). It is the author's version of a work that was accepted for publication in:

leee Transactions on Ultrasonics Ferroelectrics and Frequency Control (ISSN: 0885-3010)

\author{
Citation for the published paper: \\ Vorobiev, A. ; Gevorgian, S. (2015) "Composite Ferroelectric FBARs That Are Switchable \\ Between the First and Second Harmonics: Experimental Demonstration". Ieee Transactions \\ on Ultrasonics Ferroelectrics and Frequency Control, vol. 62(3), pp. 565-575.
}

http://dx.doi.org/10.1109/tuffc.2014.006501

Downloaded from: http://publications.lib.chalmers.se/publication/215527

Notice: Changes introduced as a result of publishing processes such as copy-editing and formatting may not be reflected in this document. For a definitive version of this work, please refer to the published source. Please note that access to the published version might require a subscription. 


\title{
Composite Ferroelectric FBARs That Are Switchable Between the First and Second Harmonics: Experimental Demonstration
}

\author{
Andrei Vorobiev and Spartak Gevorgian, Fellow, IEEE
}

\begin{abstract}
Digital switching between the first and second harmonics, in a composite thin-film bulk acoustic wave resonator (FBAR), is demonstrated experimentally. The FBAR consists of two 180-nm-thick paraelectric-phase $\mathrm{Ba}_{0.25} \mathrm{Sr}_{0.75} \mathrm{TiO}_{3}$ films separated by a 50-nm-thick $\mathrm{SrRuO}_{3}$ conducting layer. The resonant frequency of this composite resonator (with $\mathrm{Pt}$ bottom and $\mathrm{Al}$ top electrodes) is switched from $3.6 \mathrm{GHz}$ to 7.6 $\mathrm{GHz}$, where the polarity of the $5 \mathrm{~V}$ dc bias is reversed on one of the ferroelectric films. The frequency switching ratio $\left(f_{2} / f_{1}\right.$ $\geq 2$ ) depends on the thickness of the electrodes. Some adjustment of $f_{2} / f_{1}$ is possible by changing the applied dc bias.
\end{abstract}

\section{INTRODUCTION}

$\mathrm{I}^{\mathrm{s}}$ NTRINSICALLY switchable and tunable FBARs utilize dc electric-field $(E)$-induced piezoelectric effect in paraelectric-phase ferroelectric films [1]. In these films, the piezoelectric coefficient depends on the magnitude and sign of the applied dc field, $E$ :

$$
e=2\left(\varepsilon_{\mathrm{o}} \varepsilon\right)^{2} q E
$$

where $q$ is the linear electrostriction coefficient, $\varepsilon_{\mathrm{O}}$ is the dielectric constant of vacuum, and $\varepsilon$ is the dielectric permittivity. In the simplest case, the tunable FBAR consists of a paraelectric film sandwiched between two parallelplate electrodes. An applied dc voltage turns (switches) the piezoelectric effect on and transforms the parallelplate capacitor into a FBAR. The resonant, $f_{\mathrm{r}}$, and antiresonant, $f_{\mathrm{a}}$, frequencies of this $\mathrm{FBAR}$ may be controlled by the field-dependent piezoelectric coefficient $e(1)$. In contrast to standard piezoelectrics, the sign of the piezoelectric coefficient (1) may be reversed by changing the polarity of the applied dc voltage. This feature of the induced piezoelectric effect is used in frequency-switchable FBARs. In FBARs that have a single-layer ferroelectric film, the tunability of the resonant frequency is limited by the intrinsic tunability of the ferroelectric film. Greater changes are achieved in the composite FBARs consisting of two ferroelectric layers by manipulating the signs of the induced piezoelectric coefficients (1) in the ferroelectric layers [2]-[4]. Recently, a 1-D numerical model was used

Manuscript received May 8, 2014; accepted January 9, 2015.

The authors are with the Department of Microtechnology and Nanoscience, Chalmers University of Technology, 412 96, Gothenburg, Sweden (e-mail: spartak.gevorgian@chalmers.se).

DOI http://dx.doi.org/10.1109/TUFFC.2014.006501 to demonstrate switching from the first to the second harmonic in a $\mathrm{Pt} / \mathrm{SrTiO}_{3} / \mathrm{Pt} / \mathrm{SrTiO}_{3} / \mathrm{Pt}$ multilayer structure [2]. A closed-form analytic formula was proposed [3] for the dc-bias-dependent impedance of a composite FBAR that consists of two layers of paraelectric-phase ferroelectrics. In this model, the ferroelectric layers may have different thicknesses. This model is valid for resonators for which the thicknesses of the electrodes are much smaller than the thicknesses of the ferroelectric layers. The possibility of switching between the first and second harmonics was demonstrated experimentally in a stack of two $0.35-\mathrm{mm}$ thick $0.9 \mathrm{~Pb}\left(\mathrm{Mg}_{1 / 3} \mathrm{Nb}_{2 / 3}\right) \mathrm{O}_{3}-0.1 \mathrm{PbTiO}_{3}$ (0.9PMN-0.1PT) ceramic plates [4]. This work reports on frequency-switchable composite resonators based on two paraelectric phase $\mathrm{Ba}_{0.25} \mathrm{Sr}_{0.75} \mathrm{TiO}_{3}$ thin films.

\section{Test Structures and Measurement Procedure}

The cross-sectional structure of the mesa FBARs is shown in Fig. 1. Fig. 2(a) shows a photo of a substrate fragment of the fabricated mesa FBARs and the photo in Fig. 2(b) shows the details of the resonator.

The diameter of the circular mesa FBAR, Fig. 1 and Fig. 2(b), is $40 \mu \mathrm{m}$. It is arranged on a W(240 nm)/ $\mathrm{SiO}_{2}(280 \mathrm{~nm})$ Bragg reflector on a high-resistivity silicon substrate, Fig. 1. The stack of bottom electrode consists of a 110-nm-thick Pt and a 60/10-nm-thick $\mathrm{TiO}_{2} / \mathrm{Ti}$ adhesion layer. The top electrode consists of a 20/90/10-nmthick $\mathrm{Au} / \mathrm{Al} / \mathrm{Ti}$ stack. Although the tunability of the high-Ba-content paraelectric-phase $\mathrm{Ba}_{0.5} \mathrm{Sr}_{0.5} \mathrm{TiO}_{3}$ is higher [5], for frequency switching applications considered in this work, a low-Ba-content $\mathrm{Ba}_{0.25} \mathrm{Sr}_{0.75} \mathrm{TiO}_{3}$ is used because it provides a higher $Q$-factor. The $\mathrm{Ba}_{0.25} \mathrm{Sr}_{0.75} \mathrm{TiO}_{3}$ (BSTO) layers are $180 \mathrm{~nm}$ thick and the middle electrode is made of 50-nm-thick $\mathrm{SrRuO}_{3}$ (SRO) that is used for the dc bias. The large area BSTO/SrRuO/BSTO rectangular mesa stack is connected with the circular resonator mesa using a narrow neck, as shown in Fig. 2. A conducting layer [dc pad, Fig. 1, Fig. 2(a)] is deposited on its surface and the Pt ground plane under this mesa is removed (to avoid capacitive coupling between the SRO film and the ground plane). The design of the resonator allows for individual and independent dc biasing of the top and bottom ferroelectric layers. The BSTO films are produced using a pulsed laser deposition process. The details of the fabrication process are reported in [6]. 


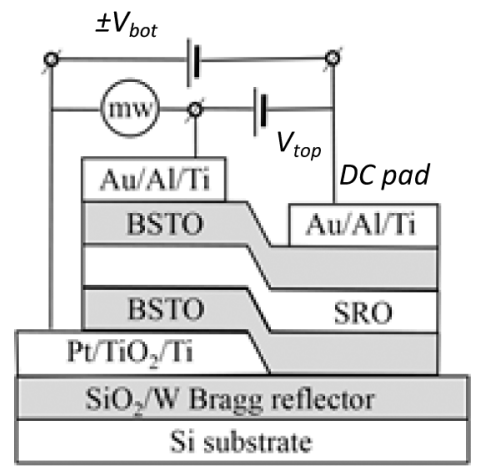

Fig. 1. Cross-sectional structure of the composite FBAR.

The simplified measurement setup is shown in Fig. 3(a). The dc bias of the middle electrode is provided by capacitive coupling using the dc pad, Fig. 1, Fig. 2. The area of the dc pad is much larger than the areas of the resonator's top and middle electrodes. Thus, the capacitance between the dc pad and middle electrode is much higher than the capacitances between the top and bottom layers of the resonator. In the dc bias circuits, Fig. 1 and Fig. 3(a), this large capacitance is connected in series with the capacitances of the top and bottom layers of the resonator. As a result, the dc bias drop on this capacitor is negligible, meaning that the dc pad is effectively connected with the middle SRO electrode. Because the leakage currents in the ferroelectric layers are negligible, the potential distribution over the whole area of the SRO middle electrode is practically uniform. The bottom and top electrodes of the resonator are used as the terminals for the one-port microwave measurements, Fig. 3(a).

In measurements, the top ferroelectric layer is always positively biased, Fig. 1, Fig. 3, whereas the dc bias of the bottom ferroelectric layer is changed from positive to negative. In the case of the first harmonic, the dc biases of the top and bottom ferroelectric layers have the same sign, Fig. 3(b). The dc-bias-induced piezoelectric coefficients (1) also have the same sign and the superimposed micro- wave signals generate acoustic waves that are in-phase in both layers. In the case of the second harmonic, the dc bias on the bottom layer is reversed, Fig. 3(c). The sign of the induced piezoelectric coefficient and the phase of the acoustic waves generated in this layer become reversed. This is regarded as anti-phase dc biasing. It is regarded as balanced if the generated acoustic waves are $180^{\circ}$ out of phase, have the same amplitude, and cancel each other. In other words, the first (odd) harmonic is prohibited.

The measured in-phase and anti-phase dc-biased complex reflection coefficients, Fig. 4(a), are used to calculate the impedance of the device under test (DUT), $Z_{\mathrm{DUT}}=$ $Z_{\mathrm{o}}(1+S) /(1-S)$. The de-embedded impedances are defined as $Z_{\mathrm{de}}(f)=Z_{\mathrm{DUT}}(f)-R\left(f_{\max }\right)$, where $R\left(f_{\max }\right)$ is the real part of the impedance at a frequency much higher than the resonant frequency. It is the sum of the resistance of the interconnect strips and resistance of the microprobe pin/electrode contact. Figs. 4(b) and 4(c) show the real and imaginary parts of the de-embedded impedances, respectively, for in-phase and anti-phase dc bias conditions.

To process and analyze the measured data, a $Q$-function, defined as

$$
Q(f)=\frac{f}{2} \frac{\partial \phi_{z}}{\partial f}
$$

is used, where $\phi_{z}$ is the impedance phase of the FBAR. In the case of the DUT, $\phi_{z}=\operatorname{atan}\left[\operatorname{Im}\left(Z_{\mathrm{DUT}}\right) / \operatorname{Re}\left(Z_{\mathrm{DUT}}\right)\right]$. For de-embedded impedances, $\phi_{z}=\operatorname{atan}\left[\operatorname{Im}\left(Z_{\mathrm{de}}\right) / \operatorname{Re}\left(Z_{\mathrm{de}}\right)\right]$. Fig. 5 represents the typical (measured and de-embedded) $Q$-functions of an FBAR in the vicinities of the first and second harmonic frequencies. The peaks of the $Q$-functions represent the resonant frequencies and the related $Q$-factors: $Q_{\mathrm{r}}=Q_{\text {peak }}=Q\left(f_{\mathrm{r}}\right)$. The minima represent the antiresonant frequencies and the related $Q$-factors: $Q_{\mathrm{a}}=$ $\operatorname{abs}\left[Q_{\min }\right]=Q\left(f_{\mathrm{a}}\right)$. Alternatively, especially for the low $Q$-factor resonators, the resonant and antiresonant frequencies are defined as frequencies where the real parts of admittance and impedance peak. The insets in Fig.

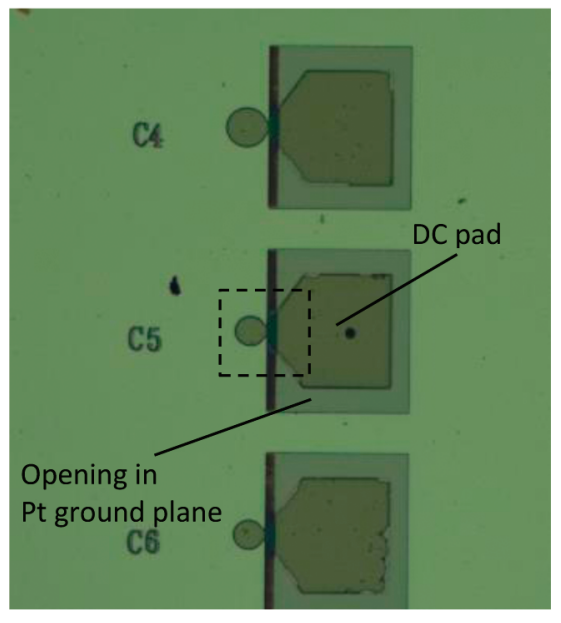

(a)

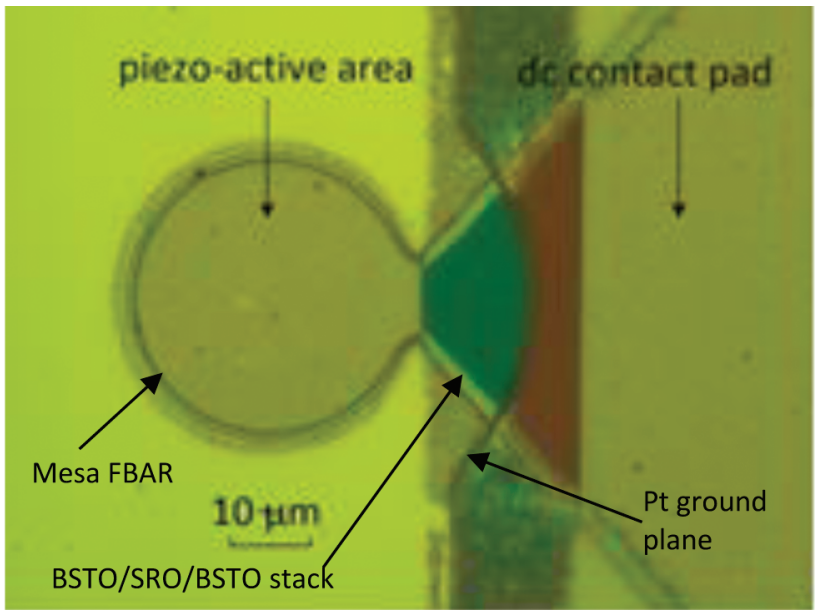

(b)

Fig. 2. (a) A fragment of the FBAR array and (b) details of the zoomed dashed box area. 


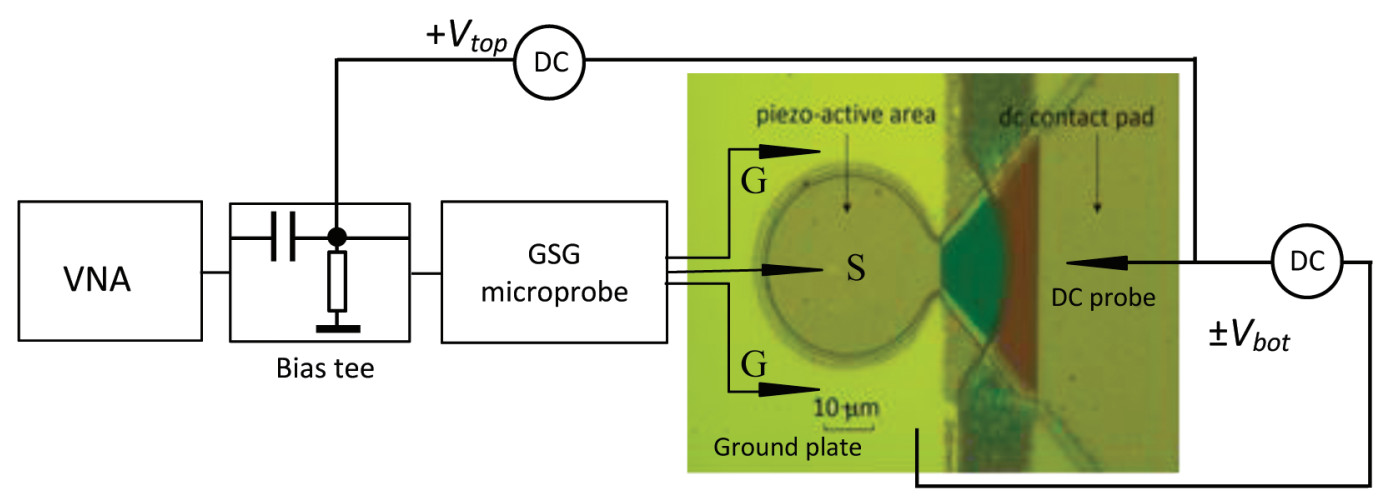

(a)

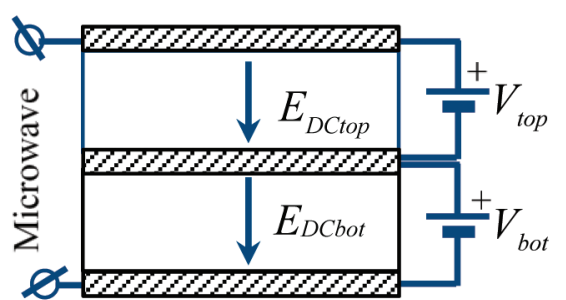

(b)

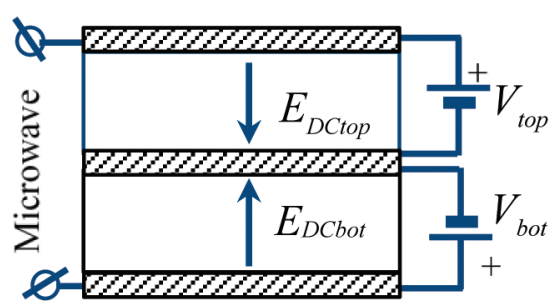

(c)

Fig. 3. (a) A simplified schematic diagram of the measurement setup, and (b) in-phase and (c) anti-phase dc bias arrangements.

5 represent the measured $Q$-functions in wider frequency ranges. As seen, only the first, Fig. 5(a), and second, Fig. 5 (b), harmonics are excited. The low- and high-frequency edges of the Bragg reflector are at about $2.5 \mathrm{GHz}$ and $8.5 \mathrm{GHz}$, respectively. Hence, the measured and simulated $Q$-factors close to these frequencies and beyond this frequency interval should be treated with caution.

\section{EXPERIMENTAL Results}

\section{A. Switching and DC-Bias-Dependent Parameters}

First consider the dc-bias dependences of the resonant/ antiresonant frequencies and the related de-embedded $Q$-factors of the composite resonator where the dc bias voltages applied to the top and bottom layers are equal in magnitude, Fig. 6. At zero bias, very weak or no resonances are detected in the measurements. For this reason, the parameters of the resonators at zero dc bias are not considered. The impedances for $5 \mathrm{~V}$ dc bias are shown in Figs. 4(b) and 4(c). In the case of in-phase dc bias (right-hand sides in Fig. 6), positive voltages of the same magnitude applied to the top and bottom layers ( $V_{\mathrm{bot}}=$ $\left.V_{\text {top }}\right)$ increase simultaneously from 0 to $+5 \mathrm{~V}$. Under a superimposed microwave voltage, both layers expand and shrink in phase, i.e., the thickness of the resonator stack oscillates as if the resonator consists of a single-layer ferroelectric film. The resonant/antiresonant frequencies are defined by the total thickness of the composite resonator. Only the first (odd) harmonic resonant and antiresonant frequencies are excited. As in the case of a single-layer

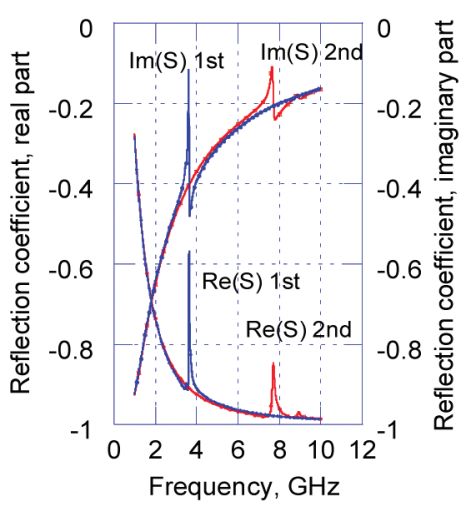

(a)

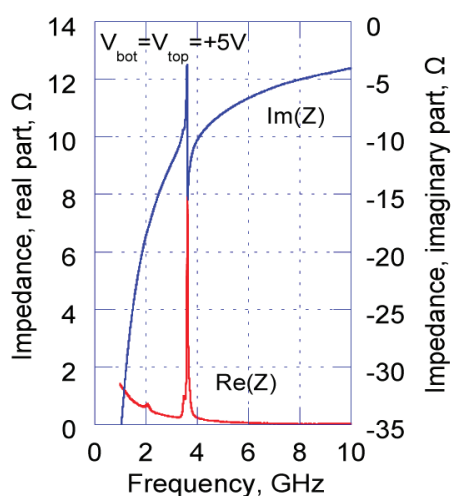

(b)

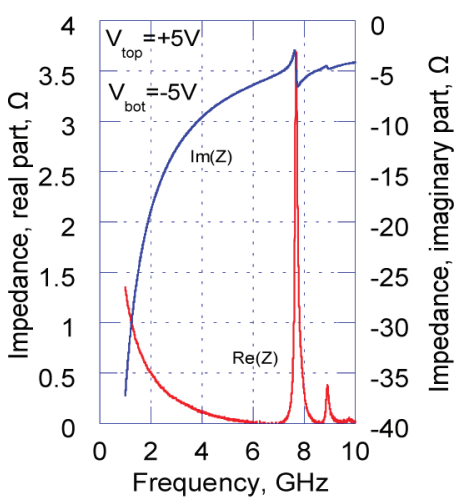

(c)

Fig. 4. Real and imaginary parts of (a) the reflection coefficients and de-embedded impedances of the measured (b) first and (c) second harmonics. For de-embedding, the series loss resistance $1.4 \Omega$ is removed from the impedance at all dc bias voltages. 


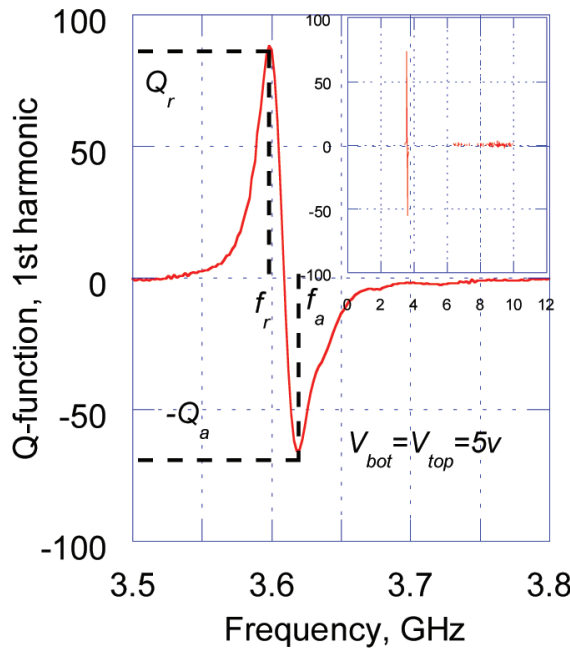

(a)

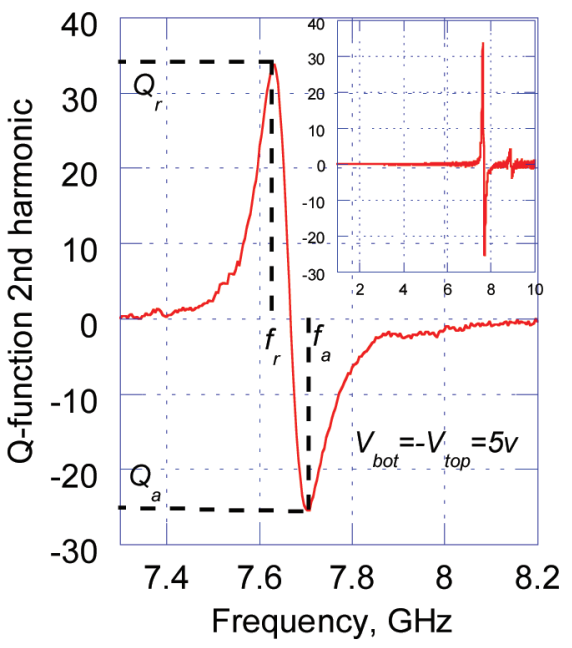

(b)

Fig. 5. Measured and de-embedded $Q$-functions of the (a) first and (b) second harmonics in the vicinity of the resonant frequencies. Insets show $Q$ functions in wider frequency ranges.

resonator, increasing the bias voltages decreases the resonant/antiresonant frequencies and increases the $Q$-factors, Fig. 6.

In the case of anti-phase dc bias (left-hand sides in Fig. 6) the polarity of the voltage applied to the bottom layer is reversed. In the top and bottom layers, the signs of the dc-field-induced piezoelectric coefficients are opposite. Under a superimposed microwave voltage, one of the layers expands while the other shrinks, i.e., there are no oscillations in the total thickness of the composite resonator. In other words, the first harmonic is suppressed. The resonant frequencies of the second harmonic are about two times higher compared with the first harmonic.

In this experiment, the tunabilities of the resonant/antiresonant frequencies, defined as $T(V)=100\left[\left(f_{\mathrm{r}, \mathrm{a}}(1 V)-\right.\right.$ $\left.f_{\mathrm{r}, \mathrm{a}}(V)\right] / f_{\mathrm{r}, \mathrm{a}}(1 V)$, are less than $1 \%$, Fig. $7(\mathrm{a})$. They are limited by the leakage current in the bottom ferroelectric layer and passive non-tunable electrodes (including the middle electrode). Similarly, the low coupling coefficient, $k_{t}^{2}=0.25 \pi^{2}\left(f_{\mathrm{a}}-f_{\mathrm{r}}\right) / f_{\mathrm{a}}$, is mainly due to the thick middle electrode, Fig. 7(b). Improvement of the tunabilities and coupling coefficients requires careful selection of the middle electrode material and reduction of its thickness. Further increasing the dc bias voltage, and thereby the tunabilities, is possible by adequately selecting the materials of the electrodes and buffer layers and by optimizing the design and fabrication processes.

\section{B. The Effects of Imbalance}

A complete suppression of the first harmonic is possible if the structure of the composite resonator is symmetric and the anti-phase bias voltages applied to the top and bottom layers are equal in magnitude. Any asymmetry in the structure causes a partial suppression of the first harmonic. The composite resonator considered in this work is asymmetrically loaded both from the bottom (Bragg reflector, bottom electrode) and top (top electrode). Even

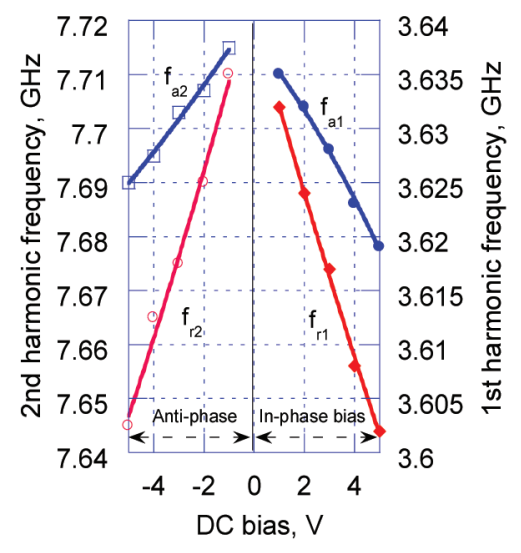

(a)

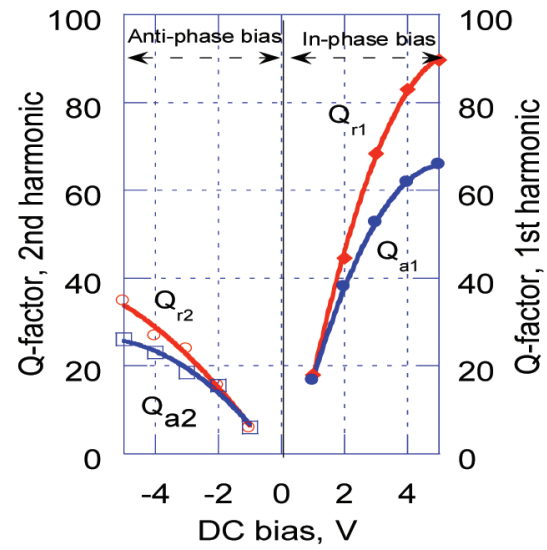

(b)

Fig. 6. Dependences of (a) the resonant/antiresonant frequencies and (b) related de-embedded $Q$-factors on the dc bias voltage. In-phase dc bias: $V_{\text {bot }}=V_{\text {top }}$, anti-phase dc bias: $V_{\text {bot }}=-V_{\text {top }}$, change from 0 to $5 \mathrm{~V}$. 


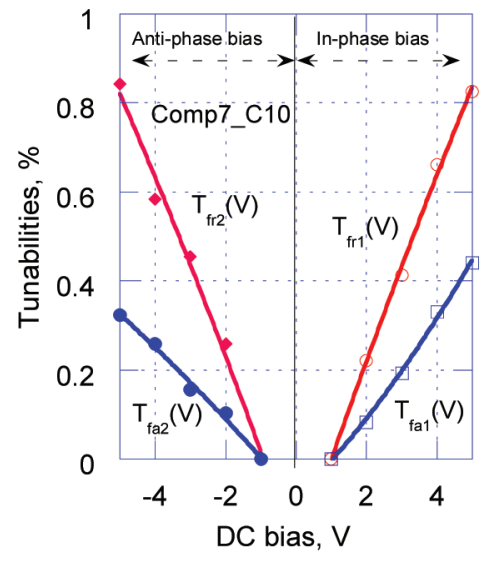

(a)

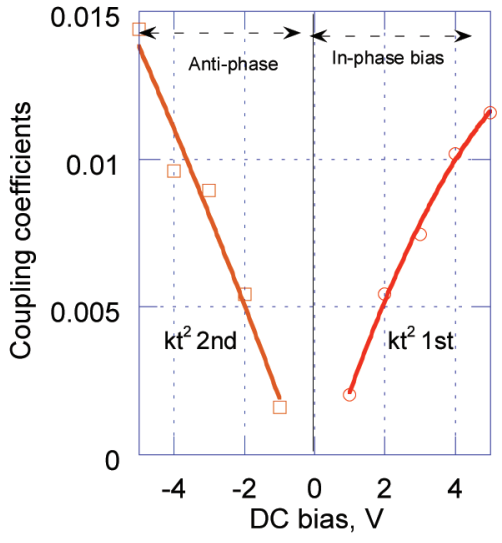

(b)

Fig. 7. Dependences of the (a) tunabilities of resonant/antiresonant frequencies and (b) coupling coefficients on the dc bias voltage.

though the ferroelectric layers are of the same thickness, their acoustic properties may differ because the bottom layer is grown on $\mathrm{Pt}$ and the top layer on $\mathrm{SrRuO}_{3}$ electrodes.

Fig. 8(a) demonstrates the incomplete suppression of the first harmonic resulting from asymmetry and imbalance in the dc bias voltages. The dc-bias dependence of the resonant/antiresonant frequencies may be used to compensate the imbalance and completely suppress the first harmonic. As an example, in Fig. 8(b), a slightly higher negative voltage is applied to the bottom layer. As seen from Fig. 8, reduction of the imbalance increases the $Q$-factors of the second harmonic.

The dependences of the resonant/antiresonant frequencies and $Q$-factors on the dc bias imbalance are shown in Fig. 9. For fixed $V_{\text {top }}=+5 \mathrm{~V}$ and $V_{\text {bot }}=0$, along with the second harmonic, the first harmonic resonances also appear. The latter is caused by the penetration of acoustic waves into the bottom layer and the reflections of these waves from the bottom and top electrode interfaces. Thus, for these extremely imbalanced anti-phase dc bias voltages, both first and second harmonics may coexist. The

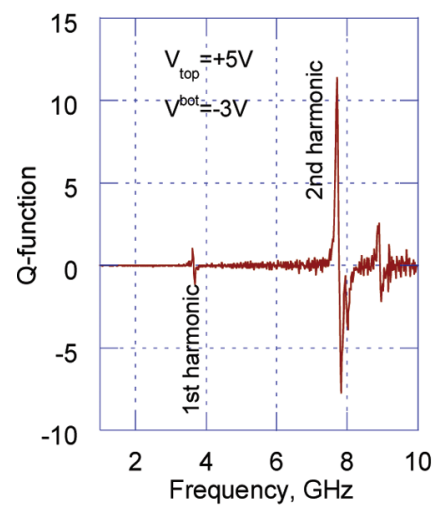

(a)

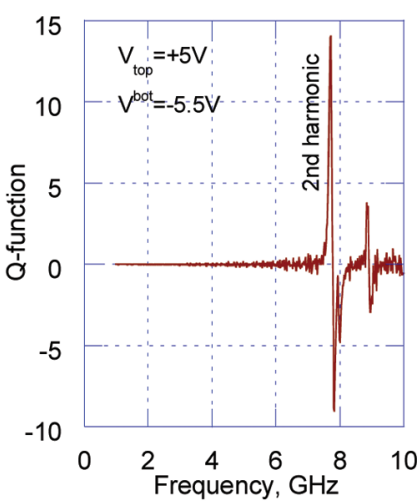

(b)
Fig. 8. Q-functions for (a) partial and (b) complete suppression of the first harmonic. gradual increase of anti-phase dc bias on the bottom layer results in reductions in the second harmonic resonant/ antiresonant frequencies and an increase in the first harmonic frequencies, Fig. 9(a). The latter is due to the constant $5 \mathrm{~V}$ dc bias applied to the top layer, which causes an electrostrictive reduction of its thickness and, hence, reduction of the total thickness of the composite. A further increase of anti-phase dc bias applied to the bottom layer increases the $Q$-factors of the second harmonic, Fig. 9(b), while the $Q$-factors of the parasitic first harmonic gradually decrease as the imbalance between the anti-phase voltages decrease. In the considered case, the $Q$-factors of the first harmonics approach to zero at approximately -3 $\mathrm{V}$ dc bias when applied to the bottom layer.

\section{Modeling and Extracting Ferroelectric Film Parameters}

In this section, a simple 1-D model of the frequencyswitchable composite resonator is considered. It is used to interpret the measured results and extract the parameters of the materials. The model may be used to optimize the performance of the resonator in the design stage, provided that the parameters of the materials are known.

\section{A. Equivalent Circuit}

Because the diameter of the resonator is much larger than the thickness, a 1-D model may be sufficient to characterize it. The proposed Mason model, implemented in an ADS circuit simulator (Keysight Technologies Inc., Santa Rosa, CA, USA), is shown in Fig. 10.

The Bragg reflector and electrode layers are represented by the ADS model of the physical transmission line [7]. The thicknesses of the layers are specified in Section II. The $Q$-factors of the films used in the model are specified in Table I. They are limited (in contrast to bulk single crystals) mainly by scattering of the acoustic waves from the grain boundaries and surface/interfacial roughness of 


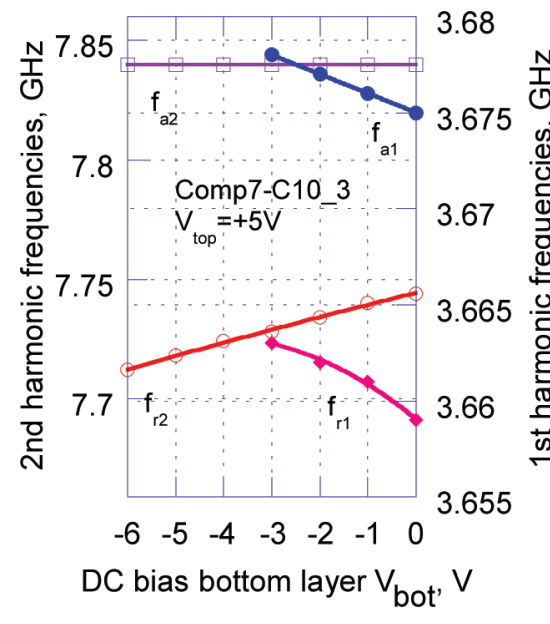

(a)

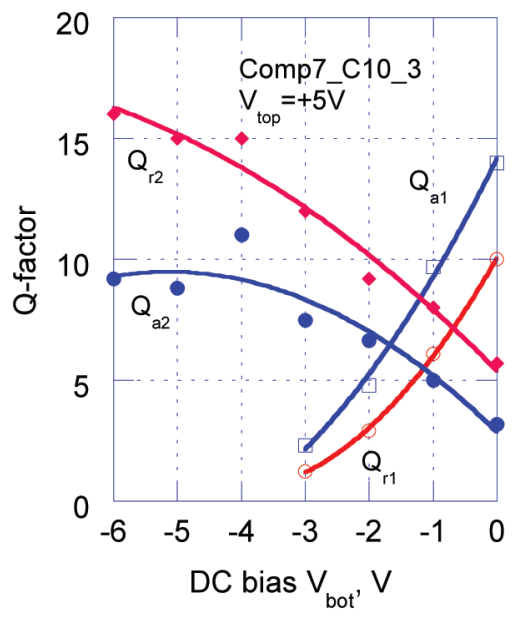

(b)

Fig. 9. Dependences of (a) the resonant/antiresonant frequencies and (b) respective de-embedded $Q$-factors on the anti-phase dc bias on the bottom layer. The dc bias on top layer is $+5 \mathrm{~V}$.

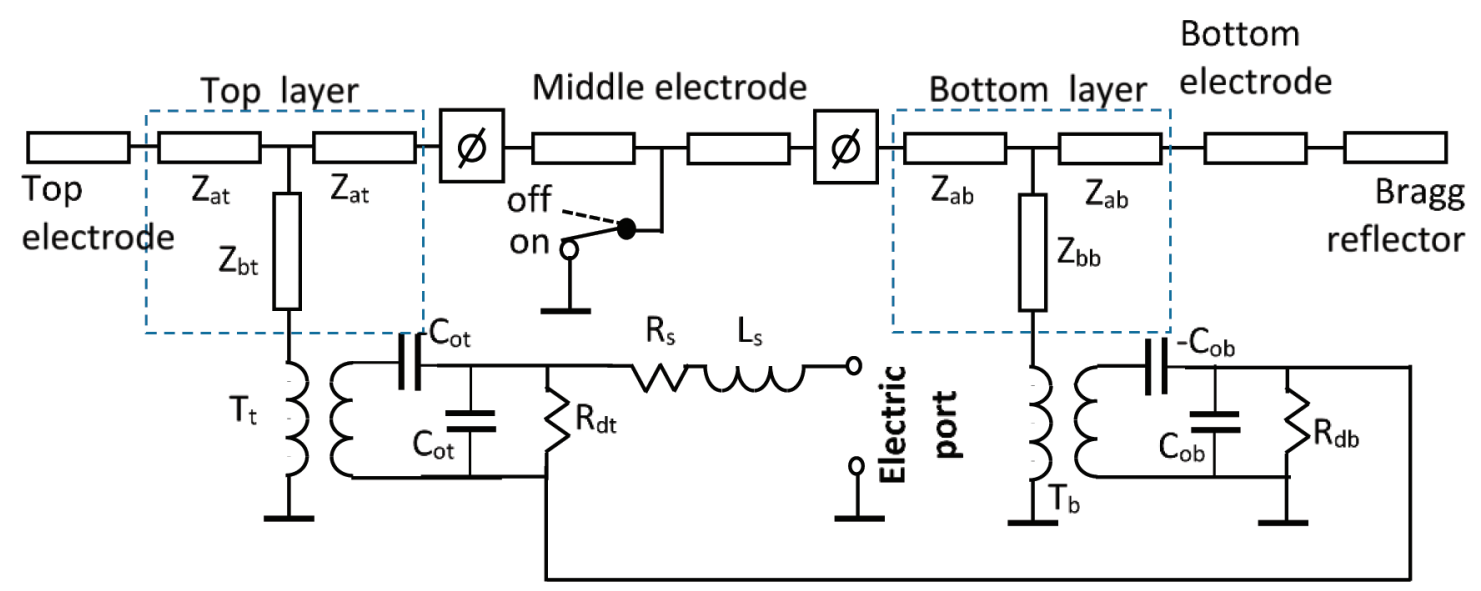

Fig. 10. Equivalent circuit of the frequency switchable composite resonator.

the films [1], [7] and strongly depend on the fabrication technology. The partial crystallization of the amorphous $\mathrm{SiO}_{2}[6]$ films causes a further reduction of the $Q$-factor because of scattering of the acoustic waves from the internal grain boundaries. For a thin $\mathrm{SiO}_{2}$ film, the attenuation and velocity at $3.6 \mathrm{GHz}$ reported in [8] are $\alpha=100 \mathrm{~cm}^{-2}$

TABLE I. Quality Factors of the ReFlector and Ferroelectric Layers (De-Embedded) Extracted From the Curve Fitting Procedure.

\begin{tabular}{lcc}
\hline & At $3.6 \mathrm{GHz}$ & At $7.2 \mathrm{GHz}$ \\
\hline$Q_{\mathrm{Al}}$ & 220 & 110 \\
$Q_{\mathrm{Au}}$ & 60 & 30 \\
$Q_{\mathrm{mech}}$ & 101 & 52 \\
$Q_{\mathrm{Pt}}$ & 80 & 40 \\
$Q_{\mathrm{Si}}$ & 1000 & 500 \\
$Q_{\mathrm{SiO} 2}$ & 200 & 100 \\
$Q_{\mathrm{SRO}}$ & 80 & 40 \\
$Q_{\mathrm{Ti}}$ & 120 & 60 \\
$Q_{\mathrm{TiO} 2}$ & 400 & 200 \\
$Q_{\mathrm{W}}$ & 50 & 25 \\
\hline
\end{tabular}

and $v_{\mathrm{ac}}=5900 \mathrm{~m} / \mathrm{s}$. The corresponding $Q$-factor, $Q=\pi$ $\cdot f /\left(\alpha \cdot v_{\mathrm{ac}}\right)=192$, is in fairly good agreement with the $Q$-factor indicated in Table I.

To extract the parameters of the resonator, the variables, such as the acoustic $Q$-factor and acoustic velocity of the ferroelectric films, are changed and the simulated impedance of the equivalent circuit is fitted against the measured impedance. The differences between the real and imaginary parts of the measured and simulated impedances are used as optimization goals. The off state of the switch, Fig. 10, corresponds to the first harmonic. The on state acoustically grounds the middle electrode (short circuit) and provides an anti-phase dc bias condition, a condition necessary but not sufficient for the complete suppression of the first harmonic. The effects of the mechanical imbalance considered in Section III-B show that the plane of the mechanical balance does not coincide with the middle of the middle electrode. To model the resonator, and to identify the plane of the mechanical balance, the middle electrode is acoustically split between the top and bottom layers (Section IV-C). 
table iI. Measured and Extracted Resonator and Material Parameters, $V=5 \mathrm{~V}$.

\begin{tabular}{lcccccccc}
\hline Harmonic & $v_{\mathrm{ac}}$ & $Q_{\text {mech }}$ & $f_{\mathrm{r}}, \mathrm{GHz}$ & $Q_{\mathrm{r}}$ & $f_{\mathrm{a}}, \mathrm{GHz}$ & $Q_{\mathrm{a}}$ & $\varepsilon$ & $Q_{\text {diel }}$ \\
\hline First measured & $\mathrm{NA}$ & & 3.603 & 89.7 & 3.617 & 65.8 & & \\
First fit & 6258 & 101 & 3.603 & 89.9 & 3.617 & 88.5 & 137 & 203 \\
Second measured & $\mathrm{NA}$ & & 7.645 & 34.7 & 7.69 & 26 & 0.013 \\
Second fit & 6198 & 52 & 7.645 & 34.6 & 7.69 & 26 & 128 & 100 \\
\hline
\end{tabular}

\section{B. First Harmonic}

As seen in Fig. 11, an excellent fit to the experimental $\mathrm{S}$-parameters is achieved. While running the optimization procedure, the measured and simulated $Q$-functions (2) are carefully monitored because the $Q$-function is the most sensitive parameter. The relatively poor matching of the $Q$-functions near the antiresonant frequency is due to dielectric and ohmic losses in the Bragg reflector layers that are not included in the model. Once a good match between measured and simulated $Q$-functions is established, Fig. 12(a), all other parameters automatically match, Figs. 12 (b) and 12(c). Neither the resonances from the second harmonic nor parasitic peaks are seen near the expected frequency range. However, a satellite peak (shoulder) is distinguishable at about $3.5 \mathrm{GHz}$, Fig. 12(b) (zoomed-in

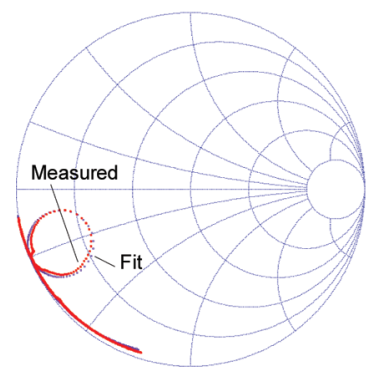

(a)

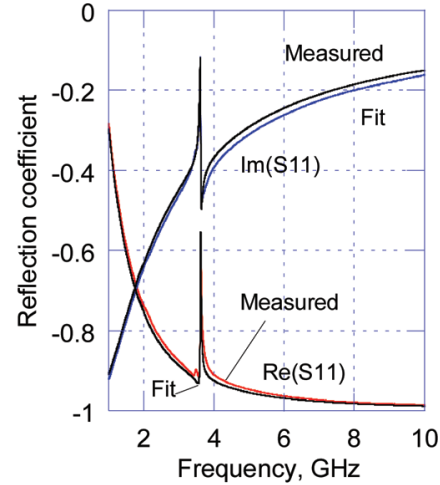

(b)

Fig. 11. First harmonic measured and fit S-parameters, $V_{\text {bot }}=V_{\text {top }}=$ $5 \mathrm{~V}$.

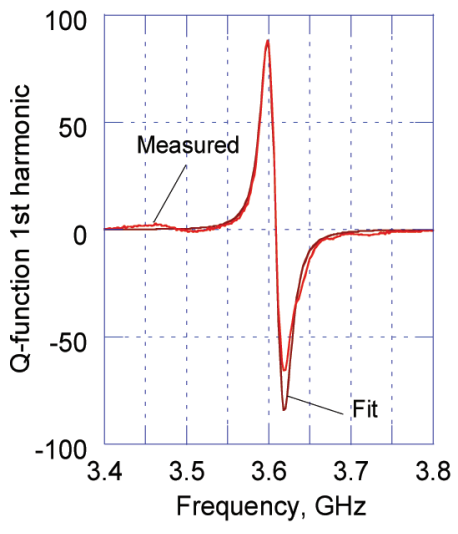

(a)

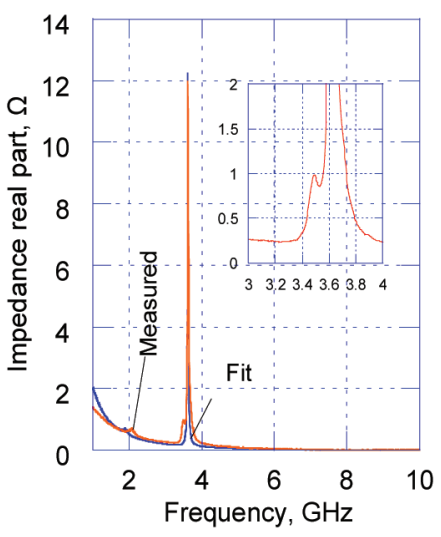

(b) inset). It is most probably an edge mode [9]. Identifying of the origin of this peak requires a $2-\mathrm{D}$ or 3 -D electromechanical modeling of the resonator. The measured and extracted parameters are given in Table II.

\section{Modeling of the Second Harmonic-Effects of the Middle Electrode}

In this case, the switch is turned on and the middle electrode is grounded electrically, Fig. 10. Ideally, the thickness of the middle electrode should be zero (if used only for dc bias). For the case considered in this work, it is rather thick $(50 \mathrm{~nm})$, almost comparable with the thicknesses of the active ferroelectric layers. If the considered structure was symmetrical, the middle electrode should have been in the minimum of the first and the maximum of the second, Fig. 13(a), harmonic displacements. The simulations show, however, that because the asymmetric loading of the resonator (from the bottom and top sides) the planes of the minimum (first harmonic) and maximum (second harmonic) displacements are shifted away (by 10 $\mathrm{nm}$ ) from the middle plane of the middle electrode, Fig. 13(b).

To take this shift into account for modeling, the middle electrode is split acoustically between the top and bottom ferroelectric layers in the equivalent circuit, Fig. 10. The splitting ratio, $m=L_{\text {middletop }} / L_{\text {middle, }}$, is selected so that the resonator layers on both sides of the splitting plane are mechanically balanced. Introducing the splitting plane is equivalent to introducing a virtual acoustic short circuit in the splitting plane. This virtual short circuit introduces extra negative phase shifts at both interfaces $\left(-L_{\text {middletop }}\right.$ - $c_{\mathrm{o}} / v_{\text {sro }}$ and $\left.-L_{\text {middlebot }} \cdot c_{\mathrm{o}} / v_{\text {sro }}, c_{\mathrm{o}}=3 \cdot 10^{8} \mathrm{~m} / \mathrm{s}\right)$. In the

Fig. 12. De-embedded measured and fit (a) first harmonic $Q$-functions, and (b) real and (c) imaginary parts of the impedance, $V_{\text {bot }}=V_{\text {top }}=5 \mathrm{~V}$. 


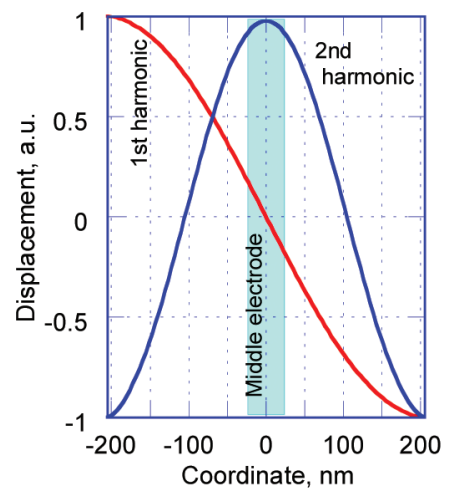

(a)

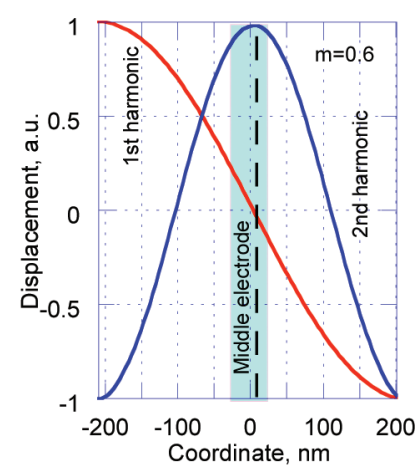

(b)
Fig. 13. Particle displacements of the first and second harmonics for (a) balanced and (b) imbalanced structures. Parameters of SRO are assumed to be identical with the parameters of the BST films and thicknesses of the top and bottom electrodes are set to be zero. The dc bias is $5 \mathrm{~V}$ for both harmonics.

equivalent circuit, Fig. 10, phase shifters $(\varnothing)$ are used to compensation for these negative phase shifts. The magnitudes of the phase shifts and the splitting ratio, $m$, are found in the curve-fitting procedure considered previously.

The real and imaginary parts of the measured and deembedded impedance and the $Q$-functions for anti-phase $\pm 5 \mathrm{~V}$ dc bias are shown in Fig. 14. As seen, the first harmonic is completely suppressed and no other features are seen around first-harmonic frequencies. However, apart from the main second harmonic, an additional damped resonance appears at about $9 \mathrm{GHz}$ (outside Bragg reflector), Fig. 14, which is associated with a large impedance contrast $\left(Z_{\mathrm{Au}} / Z_{\mathrm{Al}}=63.8 / 16.98\right)$ and reflections at the $\mathrm{Au} / \mathrm{Al}$ interface.

\section{Discussion}

\section{A. Acoustic Velocity in $\mathrm{Ba}_{0.25} \mathrm{Sr}_{0 .{ }_{5} \mathrm{O}_{3}}$ Films}

In the XRD scans, only the BST(110) orientation may be identified clearly [6]. The BST(100), (200), and (111) orientations seem to be masked by the cristobalite c(001), $\mathrm{Pt}(200)$, and $\mathrm{Pt}(111)$, respectively. For these orientations, the longitudinal acoustic wave velocities in $\mathrm{SrTiO}_{3}$ [10] are about $8000 \mathrm{~m} / \mathrm{s}$. The estimated velocity of the first harmonic $(6258 \mathrm{~m} / \mathrm{s})$ is about $20 \%$ lower, partly due to the $25 \%$ Ba content in $\mathrm{Ba}_{0.25} \mathrm{Sr}_{0.75} \mathrm{O}_{3}$, and partly due to the lower density of the films compared with the bulk singlecrystal counterparts. As seen from Table II, the acoustic velocity in the ferroelectric film decreases from 6258 $\mathrm{m} / \mathrm{s}$ (at $3.6 \mathrm{GHz}$ ) down to $6198 \mathrm{~m} / \mathrm{s}(7.6 \mathrm{GHz})$. To the best of the authors' knowledge, there is no published data on the frequency dispersion of the acoustic velocity in $\mathrm{Ba}_{x} \mathrm{Sr}_{1-x} \mathrm{O}_{3}$. For comparison, in the frequency range of 75 to $88 \mathrm{GHz}$ in [001] $\mathrm{SrTiO}_{3}$, a much higher negative slope, $-29 \mathrm{~m} /(\mathrm{s} \cdot \mathrm{GHz})$, is reported in [11]. A positive slope $(+800 \mathrm{~m} /(\mathrm{s} \cdot \mathrm{GHz})$ at $50 \mathrm{MHz}$ is reported in PZT-5A [12]. The positive slope may be explained by the second sound velocity [13]. It assumes that there is a coupling of sound to a relaxation mode and reflects the fact that the system is more rigid when probed on time scales that are short compared with the relaxation time, i.e., at higher microwave frequencies. In the framework of this theory, the frequency dependence of the sound velocity may be described by the Landau-Khalatnikov relationship [14], which may be represented as

$$
v_{\text {eff }}^{2}=v_{1}^{2}+\frac{v_{\mathrm{o}}^{2}-v_{1}^{2}}{1+(\omega \tau)^{-2}},
$$

where $\tau$ is the relaxation time and $v_{1}$ and $v_{0}$ are respectively the first and zero sound velocities. The estimated velocity around and above the second-harmonic frequencies $(f$ $>7 \mathrm{GHz}$ ) is $v_{\mathrm{eff}}=v_{\mathrm{o}}=8180 \mathrm{~m} / \mathrm{s}$. It has been shown [15] that $\mathrm{Ba}_{x} \mathrm{Sr}_{1-x} \mathrm{O}_{3}$, as a displacive ferroelectric, may have a positive slope associated with the second velocity. Hence, the measured lower slope $[-16.67 \mathrm{~m} /(\mathrm{s} \cdot \mathrm{GHz})]$ compared with $29 \mathrm{~m} /(\mathrm{s} \cdot \mathrm{GHz})$ [11], may be partly explained by the second velocity.

On the other hand, a question arises regarding whether the accuracy of the measurements is adequate for resolu-

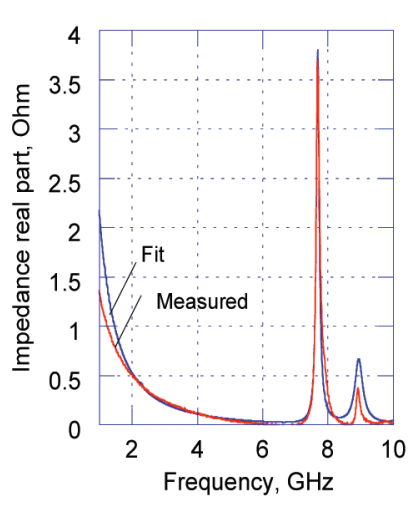

(a)

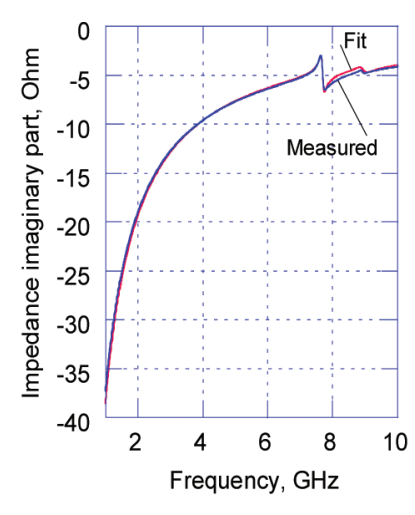

(b)

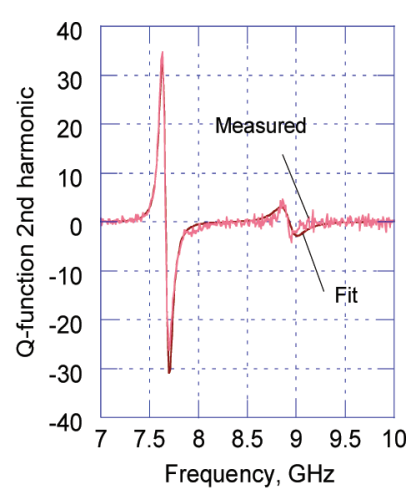

(c)

Fig. 14. De-embedded measured and fit (a) real and (b) imaginary parts of the impedance and (c) $Q$-functions of the anti-phase biased resonator. $V_{\text {bot }}=-V_{\text {top }}=5 \mathrm{~V}, m=0.6$, phase compensation is $10^{\circ}$. 


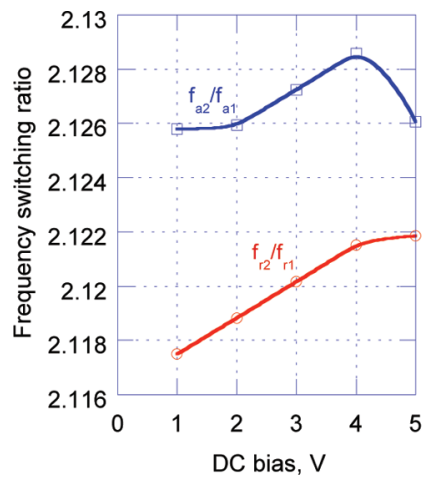

(a)

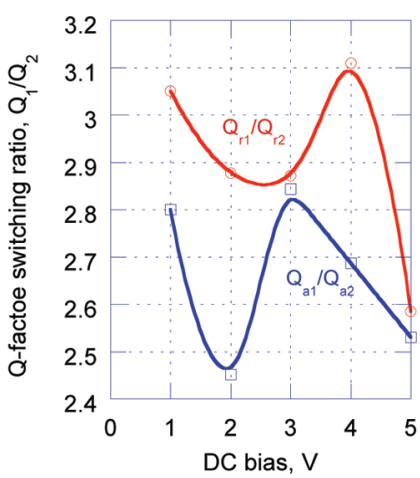

(b)
Fig. 15. Measured dependences of (a) the frequency and (b) Q-factor switching ratios on dc bias.

tion of the frequency dispersion $[-16.67 \mathrm{~m} /(\mathrm{s} \cdot \mathrm{GHz})]$ of the velocity. First, it is believed that the difference in the acoustic properties of the top and bottom ferroelectric layers is not a dominant factor. Second, by using the simple relationship for antiresonant frequency, $f_{\mathrm{a}} \approx v_{\mathrm{ac}} /(2 t)$, the relative error in the measured acoustic velocity associated with the uncertainties in the film thickness $(t)$ and frequency may be represented as $\Delta v_{\mathrm{ac}} / v_{\mathrm{ac}} \approx \Delta f / f+\Delta t / t$. Both the measurement and curve-fitting procedure contribute to the uncertainty $\Delta f$. Typically, the curve-fitting (see Table II and Figs. 11, 12, and 14) and measurement errors are less than $0.1 \%$, while the error in the thickness is greater than $1 \%$. For the typical values $t=180 \mathrm{~nm}$ and $\Delta t=5 \mathrm{~nm}$, the error in acoustic velocity is $\Delta v_{\mathrm{ac}} / v_{\mathrm{ac}}$ $\approx \Delta t / t=2.8 \%$, which is larger than the change in the velocity where the resonator is switched from the first to the second harmonic, i.e., $(6198-6258) / 258=-0.96 \%$. Thus, estimation of the frequency dispersion of the acoustic velocity is hindered by the lack of certainty in the thickness of the ferroelectric film.

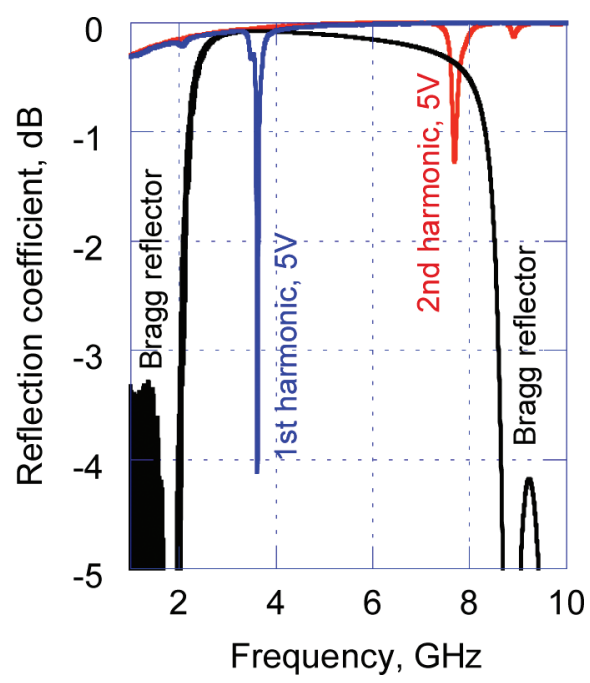

Fig. 16. Bragg reflector is leakier in the vicinity of second harmonic frequencies.

\section{B. Switching Ratios of the Resonant and Antiresonant Frequencies}

The theoretical (thicknesses of the electrodes $=0$ ) switching ratios of the resonant and antiresonant frequencies, $f_{\mathrm{r} 2} / f_{\mathrm{r} 1}$ and $f_{\mathrm{a} 2} / f_{\mathrm{a} 1}$, are equal to $2[3]$. The measured frequency switching ratios are slightly higher. They depend on the material and thicknesses of the electrodes (especially the thick middle electrode) and on the frequency dispersion of the acoustic velocity. Fig. 15(a) depicts the measured dc-bias dependences of the switching ratios of the resonant and antiresonant frequencies. Under dc bias $5 \mathrm{~V}$, the switching ratios of the resonant and antiresonant frequencies are, respectively, 2.1218 and $2.126 \mathrm{GHz}$. Some adjustment in the switching ratio is possible by tuning the dc bias, Fig. 15.

\section{Q-Factors}

In Mason's model, Fig. 10, the dielectric losses in the ferroelectric films are taken into account via loss resistors in parallel with the capacitances $C_{\mathrm{o}}$. Theoretically, both the mechanical and dielectric $Q$-factors (associated with the major loss mechanisms) are inversely proportional to the frequency, i.e., the $Q \cdot f$ product is constant [16]. Hence, one expects $Q$-factors two times smaller at resonant and antiresonant frequencies of the second harmonic.

Extracted from the curve-fitting procedure, the mechanical $Q$-factors $\left(Q_{\text {mech }}\right)$ of the ferroelectric film at resonant frequencies of the first and second harmonics are 101 and 52, respectively, Table II. In the case of the first harmonic, the difference between $Q_{\text {mech }}$ and measured $Q$ factor of the resonator $Q_{\mathrm{r} 1}(89.9)$ is about $10 \%$. In the case of the second harmonic, the difference is larger, 52 versus 35.7 , i.e., $33 \%$, Table II. The reason for this low $Q$ factor is leakage of the acoustic energy via Bragg reflector because the resonant frequency of the second harmonic, $7.645 \mathrm{GHz}$, is rather close to the high-frequency edge of the Bragg reflector, Fig. 16.

At antiresonant frequencies of the first and second harmonics, the measured and de-embedded $Q$-factors of the resonator are, respectively, 65.8 and 26, Table II. These $Q$ factors may be estimated by using the mechanical $\left(Q_{\text {mech }}\right)$ and dielectric $\left(Q_{\mathrm{d}}\right) Q$-factors of the ferroelectric films: $Q_{\mathrm{a}}$ $=\left(1 / Q_{\text {mech }}+1 / Q_{\mathrm{d}}\right)$. Using the corresponding values from Table II results in $Q_{\mathrm{a} 1}=67$ for the first harmonic, which is in fairly good agreement with the measured value of 65.8 , Table II. For the second harmonic, the calculations give $Q_{\mathrm{a} 2}=34$, which is substantially higher than the measured value of 26 , Table II. The reason for this low $Q$-factor is also due to the leakage of acoustic energy via the Bragg reflector. Interestingly, the value $Q_{\mathrm{a} 2}=34$ coincides with the measured $Q_{\mathrm{r} 2}$, Table II, i.e., without dielectric losses $Q_{\mathrm{a} 2}=Q_{\mathrm{r} 2}$. This is what one expects when the losses from series resistance and dielectric losses are small or ignored (de-embedded). 
The measured dc-bias-dependent $Q_{1} / Q_{2}$ ratios of the de-embedded $Q$-factors are in the range of 2.5 to 3, Fig. 15 (b). In this case, the higher $Q_{1} / Q_{2}$ ratio is due to the reduced $Q$-factors of the second harmonic caused by the leakage of the acoustic waves via the reflector.

\section{Conclusions}

A composite FBAR intrinsically switched between the first and second harmonics was successfully demonstrated. A 1-D ADS-based frequency-switchable Mason model of the resonator, including all the layers of the resonator and Bragg reflector, was implemented to interpret the measured results and extract the acoustic velocities and $Q-$ factors of the ferroelectric films. Under rather low dc bias voltage $(5 \mathrm{~V})$, the resonant and antiresonant frequencies are switched from about $3.6 \mathrm{GHz}$ to $7.6 \mathrm{GHz}$. The $Q-$ factors at the first harmonic frequencies are comparable with the $Q$-factors of single-layer FBARs, indicating that the $Q$-factors of SRO and BSTO are similar. They are limited by the scattering losses caused by the surface/ interface roughness of the films. $Q$-factors more than 520 (scaled from 5.2 to $3.6 \mathrm{GHz}$ ) may be achieved [17] by reducing the interfacial roughness of the films. The $\mathrm{W} / \mathrm{SiO}_{2^{-}}$ based Bragg reflector used in this experiment provides the highest reflection bandwidth. Nevertheless, the secondharmonic frequencies are close to the high-frequency edge of the reflector, where the acoustic waves leak extensively into the substrate, causing the $Q$-factors to be reduced by more than two times compared with first harmonic. Perhaps it is possible to redesign/optimize the Bragg reflector [1] and increase the $Q$-factors of the second harmonic (at the expense of somewhat reduced $Q$ factors of the first harmonic). On the other hand, increasing the $Q$-factors is possible in membrane-supported composite FBARs. Generally, the low coupling coefficients and tunabilities of the ferroelectric FBARs are due to their electrodes. In the case of composite FBARs, the extra reduction of these parameters is caused by the relatively thick middle electrode. Because the middle electrode is used only for the dc bias, its thickness may be reduced.

Theoretically, composite resonators with arbitrary frequency switching ratios, $f_{2} / f_{1}<2$, may be implemented by using more than two ferroelectric layers and internal dc bias electrodes that have zero thickness. Generally, the ferroelectric layers may have different thicknesses. This may be practically demonstrated at very low frequencies (megahertz range) where the ferroelectric layers of the resonator are much thicker than the thin film electrodes. In the case of high frequencies (gigahertz range), implementing the internal electrodes with thicknesses much smaller than the ferroelectric layer may be the main limiting factor. The surface/interface roughness of the films limits the possibility of fabricating uniform conductive/resistive layers much thinner than the ferroelectric layers. As indicated previously, the overall $Q$-factor of the resonators is also limited by the roughness of the films. Thus, the reduction of surface roughness (below 1 to $3 \mathrm{~nm}$ ) poses a major technological challenge. Apart from this, plans in the nearest future include detailed acousto-electric modeling and optimizing of the mesa structure design for higher $Q$-factors, tunabilities, and coupling coefficients.

\section{ACKNOWLEDGMENT}

The authors thank I. Gevorgyan for proofreading the manuscript.

\section{REFERENCES}

[1] S. Gevorgian, A. Tagantsev, and A. Vorobiev, Tuneable Film Bulk Acoustic Wave Resonators. London, UK: Springer, 2013.

[2] A. Kozyrev, A. Mikhailov, and S. Ptashnik, "Switchable FBAR based on paraelectric state FE films," in Proc. 40th European Microwave Conf., 2010, pp. 791-794.

[3] S. Gevorgian and A. Vorobiev, "Impedance of dc-bias-controlled composite FBARs," IEEE Trans. Ultrason. Ferroelectr. Freq. Control, vol. 60, no. 4, pp. 795-804, 2013.

[4] T. R. Gururaja, A. Shurland, and J. Chen, "Medical ultrasonic transducers with switchable frequency bands centered about $f_{\mathrm{o}}$ and $2 f_{\mathrm{o}}$, " in IEEE Ultrasonics Symp., 1997, pp. 1659-1662.

[5] A. Vorobiev and S. Gevorgian, "Enhanced tunable performance of high Q-factor $\mathrm{Ba}_{x} \mathrm{Sr}_{1-x} \mathrm{TiO}_{3}$ film bulk acoustic wave resonators," Int. J. Microw. Wireless Technol., vol. 5, no. 3, pp. 361-369, 2013.

[6] A. Vorobiev and S. Gevorgian, "Intrinsically switchable thin film bulk acoustic wave resonators," Appl. Phys. Lett., vol. 104, no. 22, art. no. 222905, 2014.

[7] S. Gevorgian, A. Vorobiev, "Loss balance in tunable ferroelectric FBARs," in Proc. European Microwave Conf., 2013, pp. 372-375.

[8] T. C. Zhu, H. J. Maris, and J. Tauc, "Attenuation of longitudinalacoustic phonons in amorphous Si02 at frequencies up to $440 \mathrm{GHz}$," Phys. Rev. B, vol. 44, no. 9, pp. 4281-4289, 1991.

[9] L. Wu, M.-C. Chure, Y.-C. Chen, K.-K. Wu and C. B. Huei, "Electrode size and dimensional ratio effect on the resonant characteristics of piezoelectric ceramic disk," in Ceramic Materials - Progress in Modern Ceramics, F. Shi, Rijeka, Croatia: Intech, 2012, ch. 2.

[10] R. O. Bell and G. Rupprecht, "Elastic constants of strontium titanate," Phys. Rev., vol. 129, pp. 90-94, 1963.

[11] S. Brivio, D. Polli, A. Crespi, R. Osellame, G. Cerullo, and R. Bertacco, "Observation of anomalous acoustic phonon dispersion in $\mathrm{SrTiO}_{3}$ by broadband stimulated Brillouin scattering," Appl. Phys. Lett., vol. 98, no. 21, art. no. 211907, 2011.

[12] H. Wang and W. Cao, "Improved ultrasonic spectroscopy methods for characterization of dispersive materials," IEEE Trans. Ultrason. Ferroelectr. Freq. Control, vol. 48, no. 4, pp. 1060-1065, 2001.

[13] Y. H. Ren, M. Trigo, R. Merlin, V. Adyam, and Q. Li, "Generation and detection of coherent longitudinal acoustic phonons in the $\mathrm{La}_{0.67} \mathrm{Sr}_{0.33} \mathrm{MnO}_{3}$ thin films by femtosecond light pulses," Appl. Phys. Lett., vol. 90, no. 25, art. no. 251918, 2007.

[14] I. M. Khalatnikov, "Absorption and dispersion of sound in a superfluid liquid near the $\lambda$ point," Sov. Phys. JETP, vol. 30, no. 2, pp. 268-272, 1970.

[15] V. L. Gurevich and A. K. Tagantsev, "Second sound in ferroelectrics," Sov. Phys. JETP, vol. 67, pp. 206-212, Jan. 1988.

[16] S. Gevorgian and A. Vorobiev, "Loss balance in tunable ferroelectric FBARs," in Proc. European Microwave Conf., 2013.

[17] A. Vorobiev and S. Gevorgian, "Tunable thin film bulk acoustic wave resonators with improved Q-factor," Appl. Phys. Lett., vol. 96, no. 21 , art. no. $212904,2010$. 


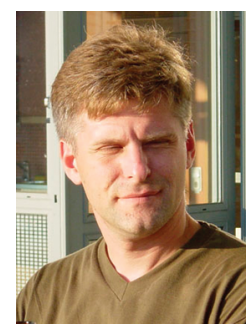

Andrei Vorobiev received his M.Sc. degree in physics of semiconductors and dielectrics from the Gorky State University, Gorky, Russia, in 1986, and his Ph.D. degree in physics and mathematics from the Institute for Physics of Microstructures of the Russian Academy of Sciences, Nizhny Novgorod, Russia, in 2000. In 2008, he received the title of Associate Professor in physical electronics from the Chalmers University of Technology, Gothenburg, Sweden. He now holds a research chair at the Chalmers University of Technology. His main research interests are in development and application of emerging functional materials and phenomena in microwave devices. His current activities focus on multiferroic and ferroelectric thin films, including development of growth processes of thin films, fabrication processes, and experimental investigation of microwave devices. Dr. Vorobiev and Dr. Gevorgian co-authored the book Ferroelectrics in Microwave Devices, Circuits and Systems. They are the inventors of patented tuneable FBARs based on the induced piezoelectric effect.

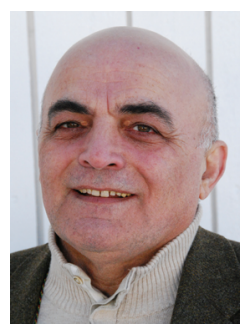

Spartak Gevorgian (M'96-SM'97-F'07) received his M.S. degree in radio engineering from the Polytechnic Institute, Yerevan, Armenia, in 1972, and his Ph.D. and Dr.Sci. degrees from the Electrical Engineering University, St. Petersburg, Russia, in 1977 and 1991, respectively. Exploration of new materials and physical phenomena for application in agile microwave devices is in the focus of his research activities, which include microwave components based on ferroelectrics, silicon RFICs and MMICs, microwave photonic devices, and modeling of passive components using conformal mapping.

Currently, he is a professor in the Department of Microtechnology and Nanoscience, Chalmers University of Technology, Sweden. He also works at Ericsson Research on a part-time basis. He has authored or co-authored more than 400 articles, papers, and patents in the fields of passive and agile microwave components, microwave photonics, and integrated optics. He and Dr. Vorobiev co-authored the book Ferroelectrics in Microwave Devices, Circuits and Systems, published by Springer, London, in 2009. His current research focuses on intrinsically switchable and tunable FBARs using ferroelectrics and multiferroics.

Prof. Gevorgian is a Member of the Academy of Sciences of Armenia. He served as a Distinguished IEEE Lecturer for the IEEE MTT-S Society for the period 2008 to 2010 . 